\title{
Role of imaging in cardiac amyloidosis: An ongoing challenge
}

\author{
Ashutosh D. Wechalekar, MD, FRCP, FRCPath, DM ${ }^{\mathrm{a}}$ \\ ${ }^{a}$ National Amyloidosis Centre, University College London (Royal Free Campus), London, United \\ Kingdom
}

Received Sep 9, 2015; accepted Sep 9, 2015

doi: $10.1007 / \mathrm{s} 12350-015-0295-0$

\section{See related article, pp.1355-1363}

Amyloidosis is a rare protein deposition disease caused by misfolding followed by tissue deposition of highly ordered aggregate proteins forming amyloid fibrils leading to progressive organ failure. ${ }^{1}$ Twenty-two different proteins have been reported to cause human disease. ${ }^{2}$ Systemic AL amyloidosis, caused by deposition of monoclonal immunoglobulin light chains, is the commonest type. Transthyretin (TTR), an inherently unstable tetrameric molecule, can deposit as amyloid fibril protein in the heart or the nerves. The wild-type molecule causes cardiac amyloidosis in the elderly (wtATTR amyloidosis or senile systemic amyloidosis) which is an increasingly recognized condition. Mutated TTR can cause familial amyloid cardiomyopathy (common in the African-American population due to deposition of variant TTR V122I) $)^{3,4}$ and much rarer autosomal dominant familial amyloid polyneuropathy due to other TTR mutations, ${ }^{5}$ some of which also cause significant cardiac amyloidosis. wtATTR amyloid deposits can be identified at autopsy in nearly a quarter of individuals over 80 years of age but the true incidence of clinically significant cardiac wtATTR amyloidosis remains unclear. ${ }^{6}$ TTR-V122I variant/ polymorphism is seen in $4 \%$ of the African-American population and the penetrance is poor. The true incidence cardiac amyloidosis remains unclear. Mutations in

Reprint requests: Ashutosh D. Wechalekar, MD, FRCP, FRCPath, DM, National Amyloidosis Centre, University College London (Royal Free Campus), Rowland Hill Street, London, NW3 2PF, United Kingdom; a.wechalekar@ucl.ac.uk

J Nucl Cardiol 2016;23:1364-7.

$1071-3581 / \$ 34.00$

Copyright (c) 2015 American Society of Nuclear Cardiology.
Apolipoprotein A1 (AApoA1) is a rare cause hereditary cardiac amyloidosis. Cardiac involvement in all types of amyloidosis is the major cause of morbidity and mortality. ${ }^{7}$

Identification of cardiac amyloid deposits is important for prognosis and planning therapy. Monitoring of these deposits is critical to assessing the adequacy of therapy, timing of next therapy, or need for ongoing therapy. The latter aspects were only of importance in systemic AL amyloidosis until the arrival of therapeutic agents, in the last few years, for treating ATTR amyloidosis (small molecules stabilizing TTR or RNA inhibitors/oligonucleotides reducing hepatic TTR synthesis). The true gold standard for defining cardiac amyloid deposition is documenting presence of these deposits on an endomyocardial biopsy. Endomyocardial biopsy is invasive, sampling errors make it unsuitable for routine serial monitoring and quantification is difficult. Furthermore, the mere presence of amyloid deposits in the heart (amyloid deposition), does not equate to these deposits causing clinically significant disease (cardiac amyloidosis). Echocardiography has been the standard method for diagnosis as well as monitoring of cardiac amyloidosis over the last two decades but is neither specific nor sensitive. ${ }^{7}{ }^{123}$ I-labeled serum amyloid $\mathrm{P}$ component (SAP) scintigraphy has been used at the UK amyloidosis center for amyloid imaging for over 20 years ${ }^{8}$ but has a major limitation due to inability to image the moving heart. Cardiac MRI (CMR) is a more discriminating method for detecting cardiac amyloidosis, but is costly, protocols are not standardized, does not differentiate types of amyloidosis and cannot be performed in renal impairment or in the presence of implanted devices. ${ }^{9}$ Bisphosphonate bone scintigraphy agents have long been reported to localize to cardiac amyloidosis. ${ }^{9}{ }^{99 \mathrm{~m}} \mathrm{Tc}$-labeled 3,3-diphosphono-1,2-propanodicarboxylic acid (DPD) ${ }^{10-12}$ and ${ }^{99 \mathrm{~m}}$ Tc-labeled pyrophosphate (PYP), ${ }^{9,13-15}$ have been systematically evaluated for imaging cardiac 
amyloidosis. ${ }^{10,16}{ }^{99 m}$ Tc-DPD or PYP are both highly sensitive for detecting cardiac ATTR amyloid deposits, even at the early/asymptomatic stage, and are now a standard part of the diagnostic algorithm for cardiac ATTR amyloidosis. The low cost, easy accessibility in any standard nuclear medicine department, few contraindications to use, and simple reporting makes both these agents attractive for identification as well as monitoring cardiac amyloid deposits and allows for standardization in assessing the extent of deposits. The molecular basis of DPD/PYP localization in the heart has not yet been determined. ${ }^{11} \mathrm{C}$-labeled Pittsburgh compound $\mathrm{B}\left({ }^{11} \mathrm{C}-\mathrm{PiB}\right){ }^{17,18}$ and ${ }^{18} \mathrm{~F}$-florbetapir (Amyvid, Eli Lilly $)^{19}$ have also been reported to be useful in cardiac amyloid imaging in a couple of small studies. ${ }^{18}$ F-florbetapir appears to be sensitive in both AL and ATTR amyloidosis but further studies are needed.

Non-invasive imaging amyloid deposits, along with concomitant use of serum biomarkers, is the only clinically relevant method of serial patient assessments allowing for standardization across institutions. Monitoring change in amyloid deposits (by imaging) is currently undertaken using echocardiography for cardiac amyloidosis and, where available, SAP scintigraphy for extra-cardiac amyloid deposits. The utility of other imaging modalities, like CMR, ${ }^{99 m}$ TcDPD/PYP scintigraphy, or ${ }^{18} \mathrm{~F}$-florbetapir, to assess amyloid progression or regression on serial imaging remains unclear. Echocardiography has significant limitations in monitoring of cardiac amyloidosis due to marked interoperator variability and poor sensitivity/specificity for determining changes, particularly subtle changes, and over time.

In this issue of the journal, Castaño and colleagues report a study of serial ${ }^{99 \mathrm{~m}}$ Tc-PYP scanning to quantify amyloid burden over time in ATTR cardiac amyloidosis. This is challenging undertaking due to the complex interaction of the tracer with competing uptake by the bone, heart, and skeletal muscle. ${ }^{99 \mathrm{~m}} \mathrm{Tc}-\mathrm{DPD}$, which essentially has the same kinetics as PYP, appears to show an early uptake by the heart and skeletal muscle followed tracer re-distribution with progressive increase in bone uptake over time with reciprocal reduction in cardiac and skeletal muscle uptake. ${ }^{20}$ In the current paper, 20 patients with cardiac ATTR (10 wild type, 10 mutant) underwent serial ${ }^{99 \mathrm{~m}}$ Tc-PYP scintigraphy. The authors report both visual semi-quantitative cardiac retention (visual score 0-no uptake to 3-uptake greater than bone) and quantitative cardiac uptake (region of interest drawn over the heart, copied, and mirrored over the contralateral chest) to calculate a heart-to-contralateral (H/CL) ratio. Both scores showed no significant change in any patient over a median 1.5 year follow-up period (visual score $3.0 \pm 0.2$ at both time points and $\mathrm{H} / \mathrm{CL}$ changed from $1.79 \pm 0.2$ to $1.73 \pm 0.2$, respectively) despite clinical progression with worsening cardiac symptoms, biomarkers, or death due to cardiac amyloidosis.

This study gives important insight into the possible natural history of cardiac ATTR amyloidosis. Cardiac ATTR amyloidosis (wtATTR or ATTR-V122I) are both considered to be slowly progressive conditions. In contrast to systemic AL amyloidosis, the natural history of this disease has not been clearly elucidated. The survival of patients with wtATTR cardiac amyloidosis is about 23 years from actual diagnosis of "cardiac amyloidosis",21_a rather dismal outcome for a supposedly slowly progressive condition. But is this the real natural history? The current study by Castaño et al reports that median uptake on ${ }^{99 \mathrm{~m}}$ Tc-PYP scintigraphy was grade 3-the maximum grade of uptake. In clinical practice, majority of the patients present with very thick walled hearts and, using CMR, amount of amyloid deposition (as evidenced by an expanded cardiac interstitial volume) is significantly greater in ATTR than AL. ${ }^{22}$ All the evidence seems to suggest that patients only present which clinical disease has advanced to a near end stage heart failure. Carriers of ATTR V122I mutation have an increased risk of incident heart failure (age- and sexstratified hazard ratio, $1.47 ; 95 \%$ CI 1.03-2.10; $P=.04)$; worse systolic and diastolic function than non-carriers even in the absence of overt manifestations of amyloid cardiomyopathy suggesting that cardiac ATTR amyloidosis (or indeed TTR amyloid interaction with other cardiac diseases) begins far earlier than traditional definitions of amyloid cardiomyopathy are reached. ${ }^{23}{ }^{99 m}$ Tc-PYP/DPD scintigraphy is highly sensitive for early detection of cardiac ATTR amyloidosis. Abnormal ${ }^{99 \mathrm{~m}}$ Tc-PYP/DPD scan is probably the very first test to show cardiac amyloid deposition in this disease. While this modality of highly discriminatory in the early stages from no amyloid to early amyloid, the saturation kinetics at higher grade uptake are unclear. It is possible that once the scan reaches grade 3 , the scans may not have the subtle discriminating characteristics needed to show a small increase in cardiac uptake. In a previous study, we noted in a few patients, the soft tissue uptake was so intense that the cardiac uptake had started to "decrease", on planar imaging. ${ }^{20}$ The authors of the present study noted a change in $\mathrm{H} / \mathrm{CL}$ ratio from $1.79 \pm 0.2$ to $1.73 \pm 0.2$ and decrease is seen in the some individual patients in Table 3 in the paper-a nonsignificant change but is this "decrease" due to increasing soft tissue uptake (the most challenging confounder for this imaging modality at higher grade uptake)? Accurate three dimensional quantification of the whole heart on cardiac uptake by SPECT-CT may make overcome some of these problems of detecting 

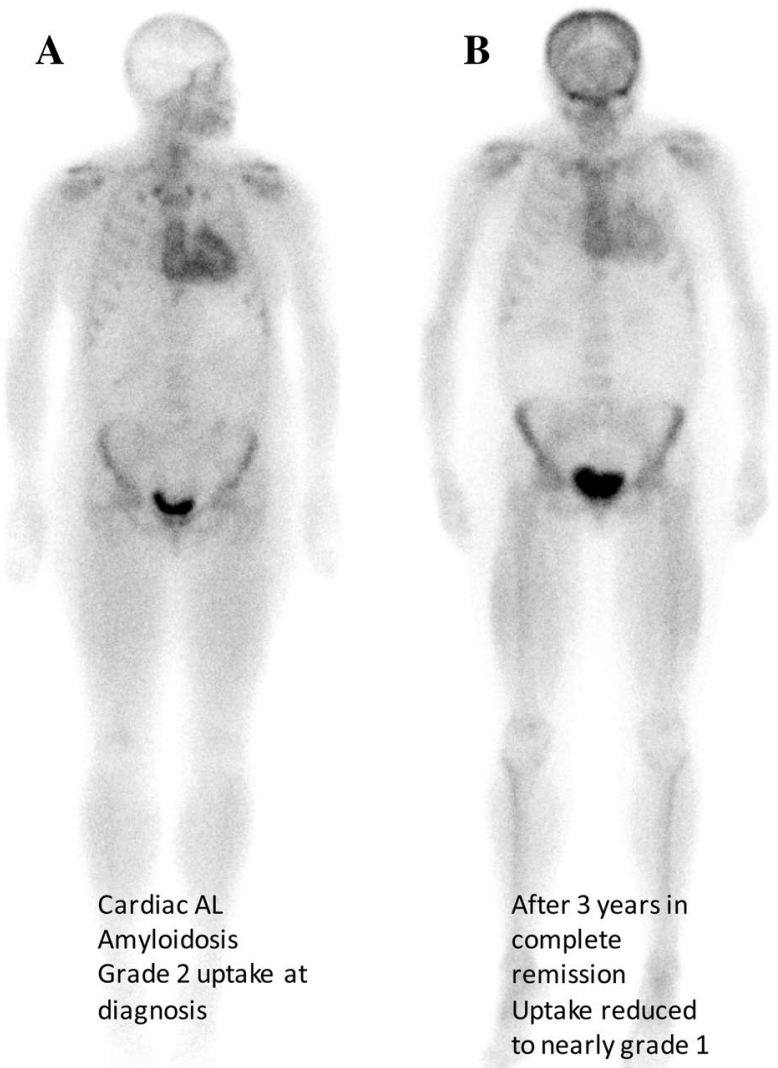

Figure 1. A patient with systemic AL amyloidosis with advanced cardiac involvement. The patient was treated with a bortezomib-based regime with a complete hematological response with improvement in cardiac biomarkers and well as functional cardiac improvement in the heart documented by 2-dimensional strain on echocardiography. There was no substantial change in the left ventricular wall thickness. A Shows the patient had a positive ${ }^{99 \mathrm{~m}} \mathrm{Tc}$-DPD scan at baseline with grade 2 cardiac uptake. The bone uptake was markedly obscured. A repeat ${ }^{99 \mathrm{~m}}$ Tc-DPD scan after 3 years, B showed a marked reduction in the cardiac uptake, reduction in the soft tissue uptake with better visualization of bones. The scan grading changed from grade 2 at baseline to nearly grade 1 at 3 years.

small changes that are not apparent on planar or singleslice SPECT-CT-based quantification.

This apparently negative study should not temper our enthusiasm for further studies to assess the utility of serial ${ }^{99 \mathrm{~m}} \mathrm{Tc}-\mathrm{PYP} / \mathrm{DPD}$ imaging in cardiac amyloidosis. We need to explore this imaging modality in early stages of the disease. Thus far, due to lack of effective treatments, the focus in ATTR has remained on imaging to assess disease progression and it is clear that the 1.5 year time scale is too short to see any progression with bisphosphonate-based imaging. Longer term studies are needed. It is possible that the changes will be more discernible if amyloid was to regress from the heart. Systemic AL amyloidosis has highly effective treatments which have improved outcomes in cardiac patients and we can document genuine cardiac improvements by biomarkers or echocardiography. Figure 1 shows a patient with advanced cardiac amyloidosis with grade 2 uptake in ${ }^{99 \mathrm{~m}} \mathrm{Tc}-\mathrm{DPD}$ scan which had improved on a repeat scan after 3 years to nearly grade 1 uptake-illustrating that ${ }^{99 \mathrm{~m}} \mathrm{Tc}-\mathrm{PYP} / \mathrm{DPD}$ scans will indeed show a change in uptake provided the is a substantial change in the amyloid burden in the patient. ${ }^{99 \mathrm{~m}}$ Tc-PYP/DPD need to be further explored to monitor cardiac AL amyloidosis where they are positive in about half of all cases. In cardiac ATTR amyloidosis, ${ }^{99 \mathrm{~m}} \mathrm{Tc}-$ PYP/DPD imaging needs assessment in patients undergoing treatment with agents which may stop amyloid deposition like Diflunisal or Tafamidis (which stabilize ATTR) and novel RNAi or antisense oligonucleotides that reduce the synthesis of precursor protein or the immunotherapeutic strategies which accelerate amyloid removal. Furthermore, the pathophysiological basis for this remarkable imaging modality still remains a mystery. Understanding this would help interpretation and refine the application of ${ }^{99 \mathrm{~m}} \mathrm{Tc}-\mathrm{PYP} / \mathrm{DPD}$ imaging in cardiac amyloidosis. Cardiac involvement in systemic AL amyloidosis has been well studied. There are established staging methods, use of biomarkers in serial assessment and criteria for defining patients with very advanced disease where the outcomes are poor irrespective the intervention barring a cardiac transplant in highly selected cases. This is lacking in ATTR amyloidosis and needs to be undertaken.

The availability of novel imaging with equilibrium CMR, the bisphosphonate imaging agents, PET scanning with agents like ${ }^{18} \mathrm{~F}$-Florbetapir, and advanced techniques in echocardiography is bringing imaging for cardiac amyloidosis into the main stream. Innovative trials integrating anti-amyloid therapies with novel imaging to assess their utility has a promise to change the outcomes in this progressive and fatal disease.

\section{Acknowledgments}

I would like to thank Mr David Hutt for the DPD scan images.

\section{References}

1. Merlini G, Bellotti V. Molecular mechanisms of amyloidosis. N Engl J Med 2003;349:583-96.

2. Sipe JD, Benson MD, Buxbaum JN, Ikeda SI, Merlini G, Saraiva MJ, et al. Nomenclature 2014: Amyloid fibril proteins and clinical classification of the amyloidosis. Amyloid 2014;21:221-4.

3. Dungu JN, Anderson LJ, Whelan CJ, Hawkins PN. Cardiac transthyretin amyloidosis. Heart 2012;98:1546-54. 
4. Connors LH, Prokaeva T, Lim A, Théberge R, Falk RH, Doros G, et al. Cardiac amyloidosis in African Americans: Comparison of clinical and laboratory features of transthyretin V122I amyloidosis and immunoglobulin light chain amyloidosis. Am Heart J 2009;158:607-14.

5. Benson MD. The hereditary amyloidoses. Best Pract Res Clin Rheumatol 2003;17:909-27.

6. Tanskanen M, Peuralinna T, Polvikoski T, Notkola IL, Sulkava R, Hardy J, et al. Senile systemic amyloidosis affects $25 \%$ of the very aged and associates with genetic variation in alpha2-macroglobulin and tau: A population-based autopsy study. Ann Med 2008;40:232-9.

7. Falk RH. Diagnosis and management of the cardiac amyloidoses. Circulation 2005;112:2047-60.

8. Hawkins PN, Lavender JP, Pepys MB. Evaluation of systemic amyloidosis by scintigraphy with 123I-labeled serum amyloid $\mathrm{P}$ component. N Engl J Med 1990;323:508-13.

9. Sachchithanantham S, Wechalekar AD. Imaging in systemic amyloidosis. Br Med Bull 2013;107:41-56.

10. Perugini E, Guidalotti PL, Salvi F, Cooke RM, Pettinato C, Riva $\mathrm{L}$, et al. Noninvasive etiologic diagnosis of cardiac amyloidosis using $\quad{ }^{99 m} \mathrm{Tc}-3,3$-diphosphono-1,2-propanodicarboxylic acid scintigraphy. J Am Coll Cardiol 2005;46:1076-84.

11. Rapezzi C, Guidalotti P, Salvi F, Riva L, Perugini E. Usefulness of Tc-99m-DPD scintigraphy in cardiac amyloidosis. J Am Coll Cardiol 2008;51:1509-10.

12. Rapezzi C, Quarta CC, Guidalotti PL, Longhi S, Pettinato C, Leone $\mathrm{O}$, et al. Usefulness and limitations of ${ }^{99 \mathrm{~m}} \mathrm{Tc}-3,3-$ diphosphono-1,2-propanodicarboxylic acid scintigraphy in the aetiological diagnosis of amyloidotic cardiomyopathy. Eur J Nucl Med Mol Imaging 2011;38:470-8.

13. Bokhari S, Castano A, Pozniakoff T, Deslisle S, Latif F, Maurer MS. (99m)Tc-pyrophosphate scintigraphy for differentiating lightchain cardiac amyloidosis from the transthyretin-related familial and senile cardiac amyloidoses. Circ Cardiovasc Imaging 2013;6:195-201.

14. Falk RH, Lee VW, Rubinow A, Skinner M, Cohen AS. Cardiac technetium- $99 \mathrm{~m}$ pyrophosphate scintigraphy in familial amyloidosis. Am J Cardiol 1984;54:1150-1.

15. Ptacin MJ. Myocardial uptake of technetium-99m pyrophosphate in systemic amyloidosis. Am J Cardiol 1984;54:938-9.

16. Puille M, Altland K, Linke RP, Steen-Müller MK, Klett R, Steiner D, et al. ${ }^{99 \mathrm{~m}} \mathrm{Tc}-\mathrm{DPD}$ scintigraphy in transthyretin-related familial amyloidotic polyneuropathy. Eur J Nucl Med Mol Imaging 2002;29:376-9.

17. Kero T, Lindsjo L, Sorensen J, Lubberink M. Accurate analysis and visualization of cardiac ${ }^{11} \mathrm{C}$-PIB uptake in amyloidosis with semiautomatic software. J Nucl Cardiol 2015. doi:10.1007/s 12350-015-0149-9.

18. Lee SP, Lee ES, Choi H, Im HJ, Koh Y, Lee MH, et al. ${ }^{11} \mathrm{C}-$ Pittsburgh B PET imaging in cardiac amyloidosis. JACC Cardiovasc Imaging 2015;8:50-9.

19. Dorbala S, Vangala D, Semer J, Strader C, Bruyere JR, Di Carli $\mathrm{MF}$, et al. Imaging cardiac amyloidosis: A pilot study using (1)(8)F-florbetapir positron emission tomography. Eur J Nucl Med Mol Imaging 2014;41:1652-62.

20. Hutt DF, Quigley AM, Page J, Hall ML, Burniston M, Gopaul D, et al. Utility and limitations of 3,3-diphosphono-1,2-propanodicarboxylic acid scintigraphy in systemic amyloidosis. Eur Heart $\mathbf{J}$ Cardiovasc Imaging 2014;15:1289-98.

21. Pinney JH, Whelan CJ, Petrie A, Dungu J, Banypersad SM, Sattianayagam P, et al. Senile systemic amyloidosis: Clinical features at presentation and outcome. J Am Heart Assoc 2013;2:e000098.

22. Fontana M, Banypersad SM, Treibel TA, Maestrini V, Sado DM, White SK, et al. Native T1 mapping in transthyretin amyloidosis. JACC Cardiovasc Imaging 2014;7:157-65.

23. Quarta CC, Buxbaum JN, Shah AM, Falk RH, Claggett B, Kitzman DW, et al. The amyloidogenic V122I transthyretin variant in elderly black Americans. N Engl J Med 2015;372:21-9. 